\title{
On Constants in Nonoscillation Criteria for Half-Linear Differential Equations
}

\author{
Simona Fišnarová and Robert Mařík \\ Department of Mathematics, Mendel University in Brno, Zemědělská 1, 61300 Brno, Czech Republic
}

Correspondence should be addressed to Simona Fišnarová, fisnarov@mendelu.cz

Received 15 June 2011; Accepted 22 August 2011

Academic Editor: Victor M. Perez Garcia

Copyright (C) 2011 S. Fišnarová and R. Mař́k. This is an open access article distributed under the Creative Commons Attribution License, which permits unrestricted use, distribution, and reproduction in any medium, provided the original work is properly cited.

We study the half-linear differential equation $\left(r(t) \Phi\left(x^{\prime}\right)\right)^{\prime}+c(t) \Phi(x)=0$, where $\Phi(x)=|x|^{p-2} x$, $p>1$. Using the modified Riccati technique, we derive new nonoscillation criteria for this equation. The results are closely related to the classical Hille-Nehari criteria and allow to replace the fixed constants in known nonoscillation criteria by a certain one-parametric expression.

\section{Introduction}

In this paper we consider the equation

$$
L[x]:=\left(r(t) \Phi\left(x^{\prime}\right)\right)^{\prime}+c(t) \Phi(x)=0,
$$

where $\Phi(x)=|x|^{p-2} x, p>1, r \in C\left(\left(t_{0}, \infty\right), \mathbb{R}^{+}\right), c \in C\left(\left(t_{0}, \infty\right), \mathbb{R}\right)$ for some $t_{0}$. Under a solution of this equation, we understand every continuously differentiable function $x$ such that $r \Phi\left(x^{\prime}\right)$ is differentiable and $(1.1)$ holds on $\left(t_{0}, \infty\right)$. This equation is called half-linear, since a constant multiple of any solution is also a solution of (1.1).

If $p=2$, then (1.1) reduces to the linear equation

$$
\left(r(t) x^{\prime}\right)^{\prime}+c(t) x=0
$$

For detailed discussion related to general theory as well as applications, we refer to [1]. According to [1], the classical linear Sturmian comparison theory extends to (1.1) and hence, if a solution has infinitely many zeros in a neighborhood of infinity, then the same is true for every solution. In this case, we say that (1.1) is oscillatory. In the opposite case, we say that 
(1.1) is nonoscillatory, as the following definition shows. Note that due to homogeneity of the set of all solutions, we can restrict ourselves to solutions which are positive in a neighborhood of infinity.

Definition 1 (nonoscillatory equation). Equation (1.1) is said to be nonoscillatory if there exist number $T \geq t_{0}$ and solution $x$ of (1.1) which satisfies $x(t)>0$ for every $t \geq T$.

Došlý and Řezníčková [2] viewed (1.1) as a perturbation of another nonoscillatory half-linear differential equation

$$
\widetilde{L}[x]:=\left(r(t) \Phi\left(x^{\prime}\right)\right)^{\prime}+\widetilde{c}(t) \Phi(x)=0
$$

and proved the following result. Note that $q$ denotes the conjugate number to $p$ in Theorem A and in the whole paper, that is, $(1 / p)+(1 / q)=1$ holds.

Theorem A (see [2, Theorem 2]). Let $h \in C^{1}$ be a positive function such that $h^{\prime}(t)>0$ for large $t$, say $t>T, \int^{\infty} r^{-1}(t) h^{-2}(t)\left(h^{\prime}(t)\right)^{2-p} \mathrm{~d} t<\infty$, and denote

$$
F_{1}(t)=\int_{t}^{\infty} \frac{\mathrm{d} s}{r(s) h^{2}(s)\left(h^{\prime}(s)\right)^{p-2}}
$$

Suppose that

$$
\begin{gathered}
\lim _{t \rightarrow \infty} F_{1}(t) r(t) h(t) \Phi\left(h^{\prime}(t)\right)=\infty \\
\lim _{t \rightarrow \infty} F_{1}^{2}(t) r(t) h^{3}(t)\left(h^{\prime}(t)\right)^{p-2} \tilde{L}[h](t)=0 .
\end{gathered}
$$

If

$$
\begin{aligned}
& \limsup _{t \rightarrow \infty} F_{1}(t) \int_{T}^{t}(c(s)-\widetilde{c}(s)) h^{p}(s) \mathrm{d} s<\frac{1}{2 q}, \\
& \liminf _{t \rightarrow \infty} F_{1}(t) \int_{T}^{t}(c(s)-\widetilde{c}(s)) h^{p}(s) \mathrm{d} s>-\frac{3}{2 q}
\end{aligned}
$$

for some $T \in \mathbb{R}$ sufficiently large, then (1.1) is nonoscillatory.

Theorem A is sharp in the sense that a convenient choice of the function $h$ allows to prove explicit sharp nonoscillation criteria. As a particular example, choosing $h(t)=$ $t^{(p-1) / p} \ln ^{2 / p} t$ and $\widetilde{c}(t)=((p-1) / p)^{p} t^{-p}$, Došlý and Ǩezníčková derived the following result for the perturbed Euler differential equation

$$
\left(\Phi\left(x^{\prime}\right)\right)^{\prime}+\left(\left(\frac{p-1}{p}\right)^{p} t^{-p}+\delta(t)\right) \Phi(x)=0
$$


Theorem B (see [2, Corollary 1]). If

$$
\begin{aligned}
& \limsup _{t \rightarrow \infty} \frac{1}{\ln t} \int^{t} \delta(s) s^{p-1} \ln ^{2} s \mathrm{~d} s<\frac{1}{2}\left(\frac{p-1}{p}\right)^{p-1}, \\
& \liminf _{t \rightarrow \infty} \frac{1}{\ln t} \int^{t} \delta(s) s^{p-1} \ln ^{2} s \mathrm{~d} s>-\frac{3}{2}\left(\frac{p-1}{p}\right)^{p-1},
\end{aligned}
$$

then (1.8) is nonoscillatory.

The constants in this criterion are optimal in some sense. Really,

$$
\liminf _{t \rightarrow \infty} \frac{1}{\ln t} \int^{t} \delta(s) s^{p-1} \ln ^{2} s \mathrm{~d} s>\frac{1}{2}\left(\frac{p-1}{p}\right)^{p-1}
$$

guarantees oscillation of (1.8) (see [2, Theorem 1]).

A variant of Theorem A without convergent integral of $r^{-1} h^{-2}\left(h^{\prime}\right)^{2-p}$ is the following.

Theorem C (see [3, Theorem 2]). Let $h \in C^{1}$ be a positive function such that $h^{\prime}(t)>0$ for large $t$, say $t>T$,

$$
F_{2}(t)=\int_{T}^{t} \frac{\mathrm{d} s}{r(s) h^{2}(s)\left(h^{\prime}(s)\right)^{p-2}}
$$

Suppose that (1.5) and (1.6) with $F_{1}$ replaced by $F_{2}$ hold. If the integral $\int^{\infty}(c(s)-\widetilde{c}(s)) h^{p}(s) \mathrm{d} s$ is convergent, and

$$
\begin{aligned}
& \limsup _{t \rightarrow \infty} F_{2}(t) \int_{t}^{\infty}(c(s)-\tilde{c}(s)) h^{p}(s) \mathrm{d} s<\frac{1}{2 q}, \\
& \liminf _{t \rightarrow \infty} F_{2}(t) \int_{t}^{\infty}(c(s)-\tilde{c}(s)) h^{p}(s) \mathrm{d} s>-\frac{3}{2 q^{\prime}},
\end{aligned}
$$

then (1.1) is nonoscillatory.

If we take $\widetilde{c}(t)=((p-1) / p)^{p} t^{-p}+(1 / 2)((p-1) / p)^{p-1} t^{-p} \ln ^{-2} t$ and $h(t)=t^{(p-1) / p} \ln ^{1 / p} t$, then Theorem $C$ can be applied to the perturbed Riemann-Weber equation

$$
\left(\Phi\left(x^{\prime}\right)\right)+\left(\left(\frac{p-1}{p}\right)^{p} t^{-p}+\frac{1}{2}\left(\frac{p-1}{p}\right)^{p-1} t^{-p} \ln ^{-2} t+\delta(t)\right) \Phi(x)=0
$$

and we obtain the following statement. 
Theorem D (see [3, Corollary 2]). If $\int_{t}^{\infty} \delta(s) s^{p-1} \ln s \mathrm{~d} s$ converges and

$$
\begin{aligned}
& \limsup _{t \rightarrow \infty} \ln (\ln t) \int_{t}^{\infty} \delta(s) s^{p-1} \ln s \mathrm{~d} s<\frac{1}{2}\left(\frac{p-1}{p}\right)^{p-1}, \\
& \liminf _{t \rightarrow \infty} \ln (\ln t) \int_{t}^{\infty} \delta(s) s^{p-1} \ln s \mathrm{~d} s>-\frac{3}{2}\left(\frac{p-1}{p}\right)^{p-1},
\end{aligned}
$$

then (1.13) is nonoscillatory.

The aim of this paper is to improve Theorems A, B, C, and D and show that the constants in the inequalities involving lim sup and lim inf can be replaced by a certain oneparametric expression. Roughly speaking, these theorems claim that the nonoscillation is preserved if the perturbation which is measured by the expressions

$$
F_{1}(t) \int_{T}^{t}(c(s)-\tilde{c}(s)) h^{p}(s) \mathrm{d} s, \quad F_{2}(t) \int_{t}^{\infty}(c(s)-\tilde{c}(s)) h^{p}(s) \mathrm{d} s
$$

is bounded in a strip between $1 / 2 q$ and $-3 / 2 q$ for large $t$. We show that there is a possibility to shift this strip down. In other words, we show that if the inequality involving limes inferior is not satisfied, it can be relaxed provided the condition involving limes superior is strengthened properly. Together with these results, we prove also similar results of a different type, where (1.1) is viewed as a standalone equation and not as a perturbation of another equation (Theorems 3.1 and 3.2).

\section{Preliminary Results}

The main tool used in the paper is the method based on the Riccati equation

$$
R[w]:=w^{\prime}+c(t)+(p-1) r^{1-q}(t)|w|^{q}=0
$$

which can be obtained from (1.1) by substitution $w=r \Phi\left(x^{\prime} / x\right)$. Our results are obtained from the following necessary and sufficient condition for nonoscillation of (1.1) which can be found, for example, in [1, Theorem 2.2.1].

Lemma 2.1. Equation (1.1) is nonoscillatory if and only if there exists a differentiable function w which satisfies the Riccati type inequality

$$
R[w](t) \leq 0
$$

for larget.

Our results heavily depend on the following relationship between the Riccati type differential operator $R[\cdot]$ and the so-called modified Riccati operator (the operator on the right-hand side of (2.3)). 
Lemma 2.2 ([4, Lemma 2.2]). Let $h$ and $w$ be differentiable functions and $v=h^{p} w-G, G=$ $\operatorname{rh} \Phi\left(h^{\prime}\right)$, then one has the identity

$$
h^{p} R[w]=v^{\prime}+h L[h]+(p-1) r^{1-q} h^{-q} H(t, v),
$$

where $H(t, v)=|v+G|^{q}-q \Phi^{-1}(G) v-|G|^{q}$

\section{Main Results}

In contrast to Theorems $\mathrm{A}$ and $\mathrm{C}$ in the first pair of theorems, we do not consider (1.1) as a perturbation of a nonoscillatory equation, but we consider this equation as a standalone problem.

Theorem 3.1. Let $h$ be a function such that $h(t)>0$ and $h^{\prime}(t) \neq 0$, both for large $t$. Suppose that the following conditions hold:

$$
\begin{gathered}
\int^{\infty} \frac{\mathrm{d} t}{r(t) h^{2}(t)\left|h^{\prime}(t)\right|^{p-2}}<\infty, \\
\lim _{t \rightarrow \infty} r(t) h(t)\left|\Phi\left(h^{\prime}(t)\right)\right| \int_{t}^{\infty} \frac{\mathrm{d} s}{r(s) h^{2}(s)\left|h^{\prime}(s)\right|^{p-2}}=\infty .
\end{gathered}
$$

If

$$
\begin{aligned}
& \limsup _{t \rightarrow \infty} \int_{t}^{\infty} \frac{\mathrm{d} s}{r(s) h^{2}(s)\left|h^{\prime}(s)\right|^{p-2}} \int^{t} h(s) L[h](s) \mathrm{d} s<\frac{1}{q}(-\alpha+\sqrt{2 \alpha}), \\
& \liminf _{t \rightarrow \infty} \int_{t}^{\infty} \frac{\mathrm{d} s}{r(s) h^{2}(s)\left|h^{\prime}(s)\right|^{p-2}} \int^{t} h(s) L[h](s) \mathrm{d} s>\frac{1}{q}(-\alpha-\sqrt{2 \alpha})
\end{aligned}
$$

for some $\alpha>0$, then (1.1) is nonoscillatory.

Proof. Denote $G:=r h \Phi\left(h^{\prime}\right), R:=r h^{2}\left|h^{\prime}\right|^{p-2}$ and

$$
v(t)=-\frac{\alpha}{q}\left(\int_{t}^{\infty} R^{-1}(s) \mathrm{d} s\right)^{-1}-\int^{t} h(s) L[h](s) \mathrm{d} s .
$$

We have

$$
\begin{aligned}
& (p-1) r^{1-q}(t) h^{-q}(t) H(t, v(t)) \\
& \quad=(p-1) r^{1-q}(t) h^{-q}(t)\left[|v(t)+G(t)|^{q}-q \Phi^{-1}(G(t)) v(t)-|G(t)|^{q}\right] \\
& \quad=(p-1) r(t)\left|h^{\prime}(t)\right|^{p}\left[\left|1+\frac{v(t)}{G(t)}\right|^{q}-q \frac{v(t)}{G(t)}-1\right] .
\end{aligned}
$$


Consider the function $F(x)=|1+x|^{q}-q x-1$. This function satisfies $F(0)=0=F^{\prime}(0)$ and $F^{\prime \prime}(0)=q(q-1)$. Hence, by the Taylor formula, the function $F(x)$ can be approximated by $(q(q-1) / 2) x^{2}$ in a neighborhood of $x=0$.

Conditions of the theorem imply that

$$
\frac{v(t)}{G(t)}=\frac{-(\alpha / q)-\int_{t}^{\infty} R^{-1}(s) \mathrm{d} s \int^{t} h(s) L[h](s) \mathrm{d} s}{G(t) \int_{t}^{\infty} R^{-1}(s) \mathrm{d} s} \longrightarrow 0 \text { as } t \longrightarrow \infty
$$

hence, for every $\varepsilon>0$, there exists $T \in \mathbb{R}$ such that

$$
F\left(\frac{v(t)}{G(t)}\right) \leq \frac{q(q-1)}{2}\left(1+\varepsilon \frac{q}{\sqrt{\alpha}}\right) \frac{v^{2}(t)}{G^{2}(t)}
$$

holds for $t>T$. At the same time $\varepsilon$ can be taken so small (will be specified later how) and $T$ so large that for $t>T$ we have

$$
\varepsilon-\frac{\alpha}{q}-\frac{\sqrt{2 \alpha}}{q}<\int_{t}^{\infty} R^{-1}(s) \mathrm{d} s \int^{t} h(s) L[h](s) \mathrm{d} s<-\frac{\alpha}{q}+\frac{\sqrt{2 \alpha}}{q}-\varepsilon .
$$

Consequently,

$$
\begin{aligned}
(p-1) r^{1-q}(t) h^{-q}(t) H(t, v(t)) & \leq \frac{q}{2}\left(1+\varepsilon \frac{q}{\sqrt{\alpha}}\right) r(t)\left|h^{\prime}(t)\right|^{p} \frac{v^{2}(t)}{G^{2}(t)} \\
& =\frac{q}{2}\left(1+\varepsilon \frac{q}{\sqrt{\alpha}}\right) \frac{\left((\alpha / q)+\int_{t}^{\infty} R^{-1}(s) \mathrm{d} s \int^{t} h(s) L[h](s) \mathrm{d} s\right)^{2}}{R(t)\left(\int_{t}^{\infty} R^{-1}(s) \mathrm{d} s\right)^{2}} \\
& <\frac{(q / 2)(1+\varepsilon(q / \sqrt{\alpha}))((\sqrt{2 \alpha} / q)-\varepsilon)^{2}}{R(t)\left(\int_{t}^{\infty} R^{-1}(s) \mathrm{d} s\right)^{2}} .
\end{aligned}
$$

Let $w=h^{-p}(v+G)$. By Lemma 2.2, we have

$$
\begin{gathered}
h^{p}(t) R[w(t)]<-\frac{\alpha}{q}\left(\int_{t}^{\infty} R^{-1}(s) \mathrm{d} s\right)^{-2} R^{-1}(t)+\frac{(q / 2)(1+\varepsilon(q / \sqrt{\alpha}))((\sqrt{2 \alpha} / q)-\varepsilon)^{2}}{R(t)\left(\int_{t}^{\infty} R^{-1}(s) \mathrm{d} s\right)^{2}} \\
=\frac{(q / 2)(1+\varepsilon(q / \sqrt{\alpha}))((\sqrt{2 \alpha} / q)-\varepsilon)^{2}-(\alpha / q)}{R(t)\left(\int_{t}^{\infty} R^{-1}(s) \mathrm{d} s\right)^{2}} .
\end{gathered}
$$


Consider the function in the numerator of the last fraction

$$
f(\varepsilon)=\frac{q}{2}\left(1+\varepsilon \frac{q}{\sqrt{\alpha}}\right)\left(\frac{\sqrt{2 \alpha}}{q}-\varepsilon\right)^{2}-\frac{\alpha}{q} .
$$

We have $f(0)=0$ and by a direct computation

$$
f^{\prime}(\varepsilon)=\frac{q}{2} \frac{q}{\sqrt{\alpha}}\left(\frac{\sqrt{2 \alpha}}{q}-\varepsilon\right)^{2}-q\left(1+\varepsilon \frac{q}{\sqrt{\alpha}}\right)\left(\frac{\sqrt{2 \alpha}}{q}-\varepsilon\right),
$$

and hence $f^{\prime}(0)=(1-\sqrt{2}) \sqrt{\alpha}<0$. This means that $\varepsilon$ can be taken so small that

$$
f(\varepsilon)<\frac{1-\sqrt{2}}{2} \varepsilon \sqrt{\alpha}
$$

Combining (3.9) and (3.12) we have

$$
h^{p}(t) R[w(t)]<\frac{((1-\sqrt{2}) / 2) \varepsilon \sqrt{\alpha}}{R(t)\left(\int_{t}^{\infty} R^{-1}(s) \mathrm{d} s\right)^{2}}<0
$$

for $t>T$ and (1.1) is nonoscillatory by Lemma 2.1.

Theorem 3.2. Let $h$ be a function such that $h(t)>0$ and $h^{\prime}(t) \neq 0$, both for large $t$. Suppose that

$$
\begin{gathered}
\int^{\infty} h(t) L[h](t) \mathrm{d} t \text { is convergent, } \\
\lim _{t \rightarrow \infty} r(t) h(t)\left|\Phi\left(h^{\prime}(t)\right)\right| \int^{t} \frac{\mathrm{d} s}{r(s) h^{2}(s)\left|h^{\prime}(s)\right|^{p-2}}=\infty .
\end{gathered}
$$

If

$$
\begin{gathered}
\limsup _{t \rightarrow \infty} \int^{t} \frac{\mathrm{d} s}{r(s) h^{2}(s)\left|h^{\prime}(s)\right|^{p-2}} \int_{t}^{\infty} h(s) L[h](s) \mathrm{d} s<\frac{1}{q}(-\alpha+\sqrt{2 \alpha}), \\
\liminf _{t \rightarrow \infty} \int^{t} \frac{\mathrm{d} s}{r(s) h^{2}(s)\left|h^{\prime}(s)\right|^{p-2}} \int_{t}^{\infty} h(s) L[h](s) \mathrm{d} s>\frac{1}{q}(-\alpha-\sqrt{2 \alpha})
\end{gathered}
$$

for some $\alpha>0$, then (1.1) is nonoscillatory.

Proof. With $R:=r h^{2}\left|h^{\prime}\right|^{p-2}$ we take

$$
v(t)=\frac{\alpha}{q}\left(\int^{t} R^{-1}(s) \mathrm{d} s\right)^{-1}+\int_{t}^{\infty} h(s) L[h](s) \mathrm{d} s
$$

and the proof is the same as the proof of Theorem 3.1. 
The following theorems are variants of Theorems 3.1 and 3.2. In these theorems we view (1.1) as a perturbation of another (nonoscillatory) equation (1.3).

Theorem 3.3. Let $h$ be a function such that $h(t)>0$ and $h^{\prime}(t) \neq 0$, both for large $t$. Suppose that (3.1) and

$$
\limsup _{t \rightarrow \infty} r(t) h^{3}(t)\left|h^{\prime}\right|^{p-2}(t) \tilde{L}[h(t)]\left(\int_{t}^{\infty} \frac{\mathrm{d} s}{r(s) h^{2}(s)\left|h^{\prime}(s)\right|^{p-2}}\right)^{2}=0
$$

hold. If

$$
\begin{aligned}
& \limsup _{t \rightarrow \infty} \int_{t}^{\infty} \frac{\mathrm{d} s}{r(s) h^{2}(s)\left|h^{\prime}(s)\right|^{p-2}} \int^{t}(c(s)-\tilde{c}(s)) h^{p}(s) \mathrm{d} s<\frac{1}{q}(-\alpha+\sqrt{2 \alpha}), \\
& \liminf _{t \rightarrow \infty} \int_{t}^{\infty} \frac{\mathrm{d} s}{r(s) h^{2}(s)\left|h^{\prime}(s)\right|^{p-2}} \int^{t}(c(s)-\widetilde{c}(s)) h^{p}(s) \mathrm{d} s>\frac{1}{q}(-\alpha-\sqrt{2 \alpha})
\end{aligned}
$$

for some $\alpha>0$, then (1.1) is nonoscillatory.

Proof. Denote $G:=r h \Phi\left(h^{\prime}\right), R:=r h^{2}\left|h^{\prime}\right|^{p-2}$ as in Theorem 3.1. Further

$$
v(t)=-\frac{\alpha}{q}\left(\int_{t}^{\infty} R^{-1}(s) \mathrm{d} s\right)^{-1}-\int^{t}(c(s)-\widetilde{c}(s)) h^{p}(s) \mathrm{d} s .
$$

Similarly as in the proof of Theorem 3.1 we get (3.4),

$$
\frac{v(t)}{G(t)}=\frac{-(\alpha / q)-\int_{t}^{\infty} R^{-1}(s) \mathrm{d} s \int^{t}(c(s)-\tilde{c}(s)) h^{p}(s) \mathrm{d} s}{G(t) \int_{t}^{\infty} R^{-1}(s) \mathrm{d} s} \longrightarrow 0 \text { as } t \longrightarrow \infty,
$$

and for sufficiently small $\varepsilon>0$, there exists $T \in \mathbb{R}$ such that

$$
(p-1) r^{1-q}(t) h^{-q}(t) H(t, v(t))<\frac{(q / 2)(1+\varepsilon(q / \sqrt{\alpha}))((\sqrt{2 \alpha} / q)-\varepsilon)^{2}}{R(t)\left(\int_{t}^{\infty} R^{-1}(s) \mathrm{d} s\right)^{2}}
$$

holds for $t>T$. Using this estimate and Lemma 2.2, we see that the function $w=h^{-p}(v+G)$ satisfies

$$
h^{p}(t) R[w(t)]<h(t) \tilde{L}[h(t)]+\frac{(q / 2)(1+\varepsilon(q / \sqrt{\alpha}))((\sqrt{2 \alpha} / q)-\varepsilon)^{2}-(\alpha / q)}{R(t)\left(\int_{t}^{\infty} R^{-1}(s) \mathrm{d} s\right)^{2}}
$$

for $t>T$. From (3.17) it follows that

$$
h(t) \tilde{L}[h(t)] R(t)\left(\int_{t}^{\infty} R^{-1}(s) \mathrm{d} s\right)^{2}<\frac{\sqrt{2}-1}{2} \varepsilon \sqrt{\alpha}
$$


for large $t$. Using this and (3.12) we get

$$
\begin{aligned}
h^{p}(t) R[w(t)] & <h(t) \tilde{L}[h(t)]+\frac{((1-\sqrt{2}) / 2) \varepsilon \sqrt{\alpha}}{R(t)\left(\int_{t}^{\infty} R^{-1}(s) \mathrm{d} s\right)^{2}} \\
& =\frac{1}{R(t)\left(\int_{t}^{\infty} R^{-1}(s) \mathrm{d} s\right)^{2}}\left[\frac{1-\sqrt{2}}{2} \varepsilon \sqrt{\alpha}+h(t) \tilde{L}[h(t)] R(t)\left(\int^{\infty} R^{-1}(t) \mathrm{d} t\right)^{2}\right] \\
& <\frac{1}{R(t)\left(\int_{t}^{\infty} R^{-1}(s) \mathrm{d} s\right)^{2}}\left[\frac{1-\sqrt{2}}{2} \varepsilon \sqrt{\alpha}+\frac{\sqrt{2}-1}{2} \varepsilon \sqrt{\alpha}\right]=0
\end{aligned}
$$

for large $t$. Hence (1.1) is nonoscillatory by Lemma 2.1 .

Corollary 3.4. If there exists $\alpha>0$ such that

$$
\begin{aligned}
& \limsup _{t \rightarrow \infty} \frac{1}{\ln t} \int^{t} \delta(s) s^{p-1} \ln ^{2} s \mathrm{~d} s<\left(\frac{p-1}{p}\right)^{p-1}(-\alpha+\sqrt{2 \alpha}), \\
& \liminf _{t \rightarrow \infty} \frac{1}{\ln t} \int^{t} \delta(s) s^{p-1} \ln ^{2} s \mathrm{~d} s>\left(\frac{p-1}{p}\right)^{p-1}(-\alpha-\sqrt{2 \alpha}),
\end{aligned}
$$

then (1.8) is nonoscillatory.

Proof. Choose $h(t)=t^{(p-1) / p} \ln ^{2 / p} t$ and $\widetilde{c}(t)=((p-1) / p)^{p} t^{-p}$. The fact that (3.1) and (3.17) hold has been proved in [2, Corollary 1]. Further,

$$
\int_{t}^{\infty} \frac{1}{r(s) h^{2}(s)\left|h^{\prime}(s)\right|^{p-2}} \mathrm{~d} s \approx\left(\frac{p}{p-1}\right)^{p-2} \frac{1}{\ln t}
$$

as shown also in [2] and the statement follows from Theorem 3.3.

Theorem 3.5. Let $h$ be a function such that $h(t)>0$ and $h^{\prime}(t) \neq 0$, both for large $t$. Suppose that the following conditions hold:

$$
\begin{aligned}
& \int^{\infty}(c(t)-\tilde{c}(t)) h^{p}(t) \mathrm{d} t \text { is convergent, } \\
& \lim _{t \rightarrow \infty} r(t) h(t)\left|\Phi\left(h^{\prime}(t)\right)\right| \int^{t} \frac{\mathrm{d} s}{r(s) h^{2}(s)\left|h^{\prime}(s)\right|^{p-2}}=\infty, \\
& \limsup _{t \rightarrow \infty} r(t) h^{3}(t)\left|h^{\prime}(t)\right|^{p-2} \tilde{L}[h(t)]\left(\int^{t} \frac{\mathrm{d} s}{r(s) h^{2}(s)\left|h^{\prime}(s)\right|^{p-2}}\right)^{2}=0 .
\end{aligned}
$$




$$
\begin{aligned}
& \limsup _{t \rightarrow \infty} \int^{t} \frac{\mathrm{d} s}{r(s) h^{2}(s)\left|h^{\prime}(s)\right|^{p-2}} \int_{t}^{\infty}(c(s)-\tilde{c}(s)) h^{p}(s) \mathrm{d} s<\frac{1}{q}(-\alpha+\sqrt{2 \alpha}), \\
& \liminf _{t \rightarrow \infty} \int^{t} \frac{\mathrm{d} s}{r(s) h^{2}(s)\left|h^{\prime}(s)\right|^{p-2}} \int_{t}^{\infty}(c(s)-\tilde{c}(s)) h^{p}(s) \mathrm{d} s>\frac{1}{q}(-\alpha-\sqrt{2 \alpha})
\end{aligned}
$$

for some $\alpha>0$, then (1.1) is nonoscillatory.

Proof. Denote $R:=r h^{2}\left|h^{\prime}\right|^{p-2}$ as in the proof of Theorem 3.1. We take

$$
v(t)=\frac{\alpha}{q}\left(\int^{t} R^{-1}(s) \mathrm{d} s\right)^{-1}+\int_{t}^{\infty}(c(s)-\widetilde{c}(s)) h^{p}(s) \mathrm{d} s,
$$

and the proof is the same as the proof of Theorem 3.3.

Corollary 3.6. If $\int_{t}^{\infty} \delta(s) s^{p-1} \ln s \mathrm{~d}$ s converges and there exists $\alpha>0$ such that

$$
\begin{aligned}
& \limsup _{t \rightarrow \infty} \ln (\ln t) \int_{t}^{\infty} \delta(s) s^{p-1} \ln s \mathrm{~d} s<\left(\frac{p-1}{p}\right)^{p-1}(-\alpha+\sqrt{2 \alpha}), \\
& \liminf _{t \rightarrow \infty} \ln (\ln t) \int_{t}^{\infty} \delta(s) s^{p-1} \ln s \mathrm{~d} s>\left(\frac{p-1}{p}\right)^{p-1}(-\alpha-\sqrt{2 \alpha}),
\end{aligned}
$$

then (1.13) is nonoscillatory.

Proof. We take $\widetilde{c}(t)=((p-1) / p)^{p} t^{-p}+1 / 2((p-1) / p)^{p-1} t^{-p} \ln ^{-2} t$ and $h(t)=t^{(p-1) / p} \ln ^{1 / p} t$. Then, as shown in the proof of [3, Corollary 2], all assumptions of Theorem 3.5 hold and

$$
\int_{t}^{\infty} \frac{1}{r(s) h^{2}(s)\left|h^{\prime}(s)\right|^{p-2}} \mathrm{~d} s \approx\left(\frac{p}{p-1}\right)^{p-2} \ln (\ln t) .
$$

Hence, the statement follows from Theorem 3.5.

In the following theorem we employ the technique used in previous results directly for Riccati operator from (2.1) rather than for modified Riccati operator from (2.3). This method yields a result which is (as far as we know) new even in the linear case (Theorem 3.7) and offers also a simple and alternative proof of known results (see Remark 6).

Theorem 3.7. Let the following conditions hold:

$$
\int^{\infty} r^{1-q}(t) \mathrm{d} t<\infty
$$


and for some $\alpha>0$

$$
\begin{aligned}
& \limsup _{t \rightarrow \infty}\left(\int_{t}^{\infty} r^{1-q}(s) \mathrm{d} s\right)^{p-1} \int^{t} c(s) \mathrm{d} s<-\alpha+\alpha^{1 / q}, \\
& \liminf _{t \rightarrow \infty}\left(\int_{t}^{\infty} r^{1-q}(s) \mathrm{d} s\right)^{p-1} \int^{t} c(s) \mathrm{d} s>-\alpha-\alpha^{1 / q} .
\end{aligned}
$$

Then (1.1) is nonoscillatory.

Proof. From the assumptions of the theorem it follows that there exist $\varepsilon$ and $T$ such that $0<$ $\varepsilon<\alpha^{1 / q}$ and

$$
-\alpha-\alpha^{1 / q}+\varepsilon<\left(\int_{t}^{\infty} r^{1-q}(s) \mathrm{d} s\right)^{p-1} \int^{t} c(s) \mathrm{d} s<-\alpha+\alpha^{1 / q}-\varepsilon
$$

for every $t>T$. Define $w(t)=-\alpha\left(\int_{t}^{\infty} r^{1-q}(s) \mathrm{d} s\right)^{1-p}-\int^{t} c(s) \mathrm{d} s$. Direct computation shows

$$
\begin{aligned}
w^{\prime}(t)+ & c(t)+(p-1) r^{1-q}(t)|w(t)|^{q} \\
= & -\alpha(p-1) r^{1-q}(t)\left(\int_{t}^{\infty} r^{1-q}(s) \mathrm{d} s\right)^{-p} \\
& +(p-1) r^{1-q}(t)\left|\alpha\left(\int_{t}^{\infty} r^{1-p}(s) \mathrm{d} s\right)^{1-p}+\int^{t} c(s) \mathrm{d} s\right|^{q} \\
= & (p-1) r^{1-q}(t)\left(\int_{t}^{\infty} r^{1-q}(s) \mathrm{d} s\right)^{-p}\left(-\alpha+\left|\alpha+\left(\int_{t}^{\infty} r^{1-q}(s) \mathrm{d} s\right)^{p-1} \int^{t} c(s) \mathrm{d} s\right|^{q}\right) \\
< & (p-1) r^{1-q}(t)\left(\int_{t}^{\infty} r^{1-q}(s) \mathrm{d} s\right)^{-p}\left(-\alpha+\left|\alpha^{1 / q}-\varepsilon\right|^{q}\right) \\
< & 0
\end{aligned}
$$

for every $t>T$. The nonoscillation of (1.1) follows from Lemma 2.1.

Corollary 3.8. For $\lambda<0$ denote by $\mu(\lambda)$ the positive root of the equation $z^{1 / q}+z+\lambda=0$. Denote

$$
\begin{gathered}
A_{*}=\liminf _{t \rightarrow \infty}\left(\int_{t}^{\infty} r^{1-q}(s) d s\right)^{p-1} \int^{t} c(s) \mathrm{d} s, \\
A^{*}=\limsup _{t \rightarrow \infty}\left(\int_{t}^{\infty} r^{1-q}(s) d s\right)^{p-1} \int^{t} c(s) \mathrm{d} s .
\end{gathered}
$$


If (3.32) holds and

$$
A_{*} \leq-\frac{2 p-1}{p}\left(\frac{p-1}{p}\right)^{p-1}, \quad A^{*}<\left(\mu\left(A_{*}\right)\right)^{1 / q}-\mu\left(A_{*}\right)
$$

then (1.1) is nonoscillatory.

Proof. It follows from the fact that $y=\mu^{1 / q}(x)-\mu(x)$ is for $x \leq-((2 p-1) / p)((p-1) / p)^{p-1}$ explicit formula for the curve given parametrically by $x=-\alpha-\alpha^{1 / q}, y=-\alpha+\alpha^{1 / q}$ for $\alpha \geq q^{-p}$. This curve is increasing for $\alpha>q^{-p}$ and (3.33) means that the point $\left(A_{*}, A^{*}\right)$ is below this curve. The same is ensured by inequalities (3.37).

\section{Concluding Remarks and Comments}

Remark 1. If we put $\alpha=1 / 2$ in Theorems 3.3 and 3.5, we get Theorems A and C. The constant $-\alpha+\sqrt{2 \alpha}$ from the condition with limes superior is maximal with this choice. As far as we know, Theorems 3.3 and 3.5 are new if $\alpha \neq 1 / 2$ and Theorems 3.1 and 3.2 are new for every $\alpha>0$. Similarly, if we put $\alpha=q^{-p}$ in Theorem 3.7, then we get [1, Theorem 3.1.5].

Remark 2. If $\alpha>1 / 2$, then both $-\alpha \pm \sqrt{2 \alpha}$ are decreasing functions of the variable $\alpha$ and thus the bounds for $F_{1}(t) \int_{T}^{t}(c(s)-\widetilde{c}(s)) h^{p}(s)$ and $F_{2}(t) \int_{t}^{\infty}(c(s)-\widetilde{c}(s)) h^{p}(s)$ d $s$ which guarantee nonoscillation in Theorems 3.3 and 3.5 also decrease. Closer investigation shows that the bound for limes inferior decreases faster and thus the maximal allowed difference between limes superior and inferior is allowed to be bigger if both are small. A similar remark applies also to the results from Theorems 3.1 and 3.2.

Remark 3. The nonoscillation criteria in the previous theorems are written in the form

$$
\limsup _{t \rightarrow \infty} f(t)<\frac{1}{q}(-\alpha+\sqrt{2 \alpha}), \quad \liminf _{t \rightarrow \infty} f(t)>\frac{1}{q}(-\alpha-\sqrt{2 \alpha}),
$$

where the definition of $f(t)$ varies for each particular theorem. Note that if inequalities (4.1) hold for some $\alpha$ which satisfies $0<\alpha<1 / 2$, then they hold also for $\alpha=1 / 2$. In view of this fact it is reasonable to suppose $\alpha \geq 1 / 2$.

Remark 4. Denote

$$
f_{*}=\liminf _{t \rightarrow \infty} f(t), \quad f^{*}=\limsup _{t \rightarrow \infty} f(t) .
$$

It is easy to show that the parametric curve $x=(1 / q)(-\alpha-\sqrt{2 \alpha}), y=(1 / q)(-\alpha+\sqrt{2 \alpha}), \alpha \geq$ $1 / 2$, is an increasing function with nonparametric equation $y=x-(2 / q)+(2 / q) \sqrt{1-2 q x}$. Since (4.1) expresses the fact that the point $\left(f_{*}, f^{*}\right)$ is below this curve, inequalities (4.1) are satisfied if

$$
f_{*} \leq-\frac{3}{2 q}, \quad f^{*}<f_{*}-\frac{2}{q}+\frac{2}{q} \sqrt{1-2 q f_{*}}
$$


Remark 5. Let us compare our results with known results on a particular example of perturbed Euler equation (1.8). Let us denote

$$
\begin{gathered}
g(t)=\left(\frac{p}{p-1}\right)^{p-1} \frac{1}{\ln t} \int^{t} \delta(s) s^{p-1} \ln ^{2} s \mathrm{~d} s, \\
g_{*}=\liminf _{t \rightarrow \infty} g(t), \quad g^{*}=\limsup _{t \rightarrow \infty} g(t),
\end{gathered}
$$

and rewrite the conditions from Corollary 3.4 into

$$
g_{*}>-\alpha-\sqrt{2 \alpha}, \quad g^{*}<-\alpha+\sqrt{2 \alpha}
$$

It is easy to see that every equation in the form (1.8) can be associated with some point in the plane $g_{*}$ and $g^{*}$. On the other hand, for every point in this plane which satisfies $g^{*} \geq g_{*}$, we can construct an equation in the form (1.8) which is associated with this point.

As mentioned before, Došlý and Řezníčková [2] proved that (1.8) is oscillatory if $g_{*}>$ $1 / 2$ and nonoscillatory if $g_{*}>-3 / 2$ and $g^{*}<1 / 2$. This gives two regions in $g_{*} g^{*}$ plane with resolved oscillation properties of (1.8): the unbounded region of oscillation has the form of the angle with vertex $[1 / 2,1 / 2]$, rays $g_{*}=1 / 2$ and $g_{*}=g^{*}$, open up and the bounded region of nonoscillation has the form of triangle with vertexes $[1 / 2,1 / 2],[-3 / 2,1 / 2]$, and $[-3 / 2,-3 / 2]$. Corollary 3.4 allows us to extend the region of nonoscillation by the unbounded region which is between the line $g^{*}=g_{*}$ and the curve given for $\alpha \geq 1 / 2$ parametrically by $g_{*}=-\alpha-\sqrt{2 \alpha}, g^{*}=-\alpha+\sqrt{2 \alpha}$ or, equivalently, given by $g^{*}=g_{*}-2+2 \sqrt{1-2 g_{*}}$ for $g_{*} \leq-3 / 2$. All these regions are shown on Figure 1.

In particular, if $\delta(t)=k((p-1) / p)^{p-1} t^{-p} \ln ^{-2}(t)$, then $g_{*}=g^{*}=k$ and (1.8) is oscillatory if $k>1 / 2$ and nonoscillatory if $k \leq 1 / 2$. This observation has been made already by Elbert and Schneider in [5] and has a close connection with the so-called conditional oscillation, see [6] for more details related to conditional oscillation.

Remark 6. If the integral $\int^{\infty} c(s) \mathrm{d} s$ is convergent and if we use the method from Theorem 3.7 and Corollary 3.8 with $w(t)=\alpha\left(\int^{t} r^{1-q}(s) \mathrm{d} s\right)^{-1}+\int_{t}^{\infty} c(s) \mathrm{d} s$, we get the second part of [1, Theorem 3.3.6], which is originally due for $p \leq 2$ to Kandelaki et al. ([7, Theorem 1.6]).

Remark 7. If $\alpha=1 / 4$ and $p=2$, then Theorem 3.7 reduces to well-known Hille-Nehari nonoscillation criteria. In this case the constants from (3.33) reduce to $1 / 4$ and $-3 / 4$.

Remark 8. If we use the additional condition

$$
\liminf _{t \rightarrow \infty} r(t) h(t) \Phi\left(h^{\prime}(t)\right)>0,
$$

then the conclusion related to that of Theorems 3.2 and 3.5 can be derived from known results. Really, denote $R=r h^{2}\left|h^{\prime}\right|^{p-2}$ and $C=h L[h]$, where $h$ is a positive function such that $h^{\prime} \neq 0$ and suppose that $\int^{\infty} R^{-1}(t) \mathrm{d} t=\infty$ and $\int^{\infty} C(t) \mathrm{d} t$ is convergent. Under these conditions, an alternative version of Theorem 3.2 can be derived using the so-called linearization technique. This technique is based on comparison of the (non)oscillation of (1.1) with that of a certain linear equation. The relation between these equations is hidden in identity (2.3) which (after 


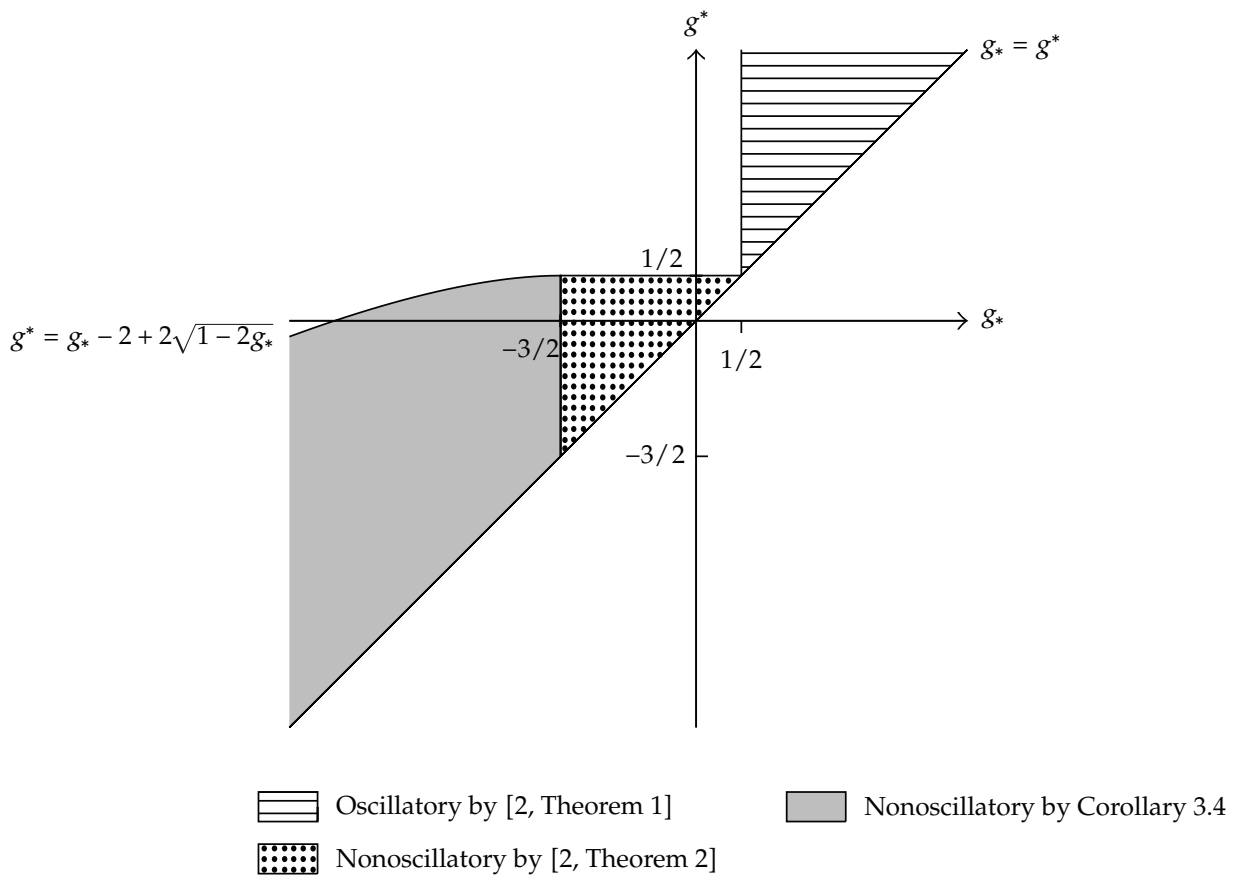

Figure 1: Regions of oscillation and nonoscillation of perturbed Euler equation.

the quadratization of the last term on the right-hand side) relates the associated Riccati operators. More precisely, nonoscillation of (1.1) is implied by nonoscillation of the linear equation

$$
\left(R(t) y^{\prime}\right)+\frac{q+\varepsilon}{2} C(t) y=0
$$

where $\varepsilon>0$ is arbitrary. Applying the linear version $(p=2)$ of the criteria discussed in Remark 6 to the above linear equation, we obtain the conditions for limes inferior and superior from Theorem 3.2. Similarly if $C=(c-\widetilde{c}) h^{p}$, where $h$ is a positive solution of (1.3), then we get the conditions for limes inferior and superior from Theorem 3.5. Note that if $p=2$, then $R(t)$ and $C(t)$ are the coefficients of the equation which results from (1.2) (the special case of (1.1) for $p=2$ ) upon the transformation $x=h y$. We refer to [8-10] for results concerning the linearization technique and for the half-linear Hille-Nehari type criteria derived using this technique from the classical criteria mentioned in Remark 7.

\section{Acknowledgment}

Research supported by the Grant P201/10/1032 of the Czech Science Foundation.

\section{References}

[1] O. Došlý and P. Řehák, Half-Linear Differential Equations, vol. 202 of North-Holland Mathematics Studies, Elsevier Science, Amsterdam, The Netherlands, 2005. 
[2] O. Došlý and J. Řezníčková, “Oscillation and nonoscillation of perturbed half-linear Euler differential equations," Publicationes Mathematicae Debrecen, vol. 71, no. 3-4, pp. 479-488, 2007.

[3] O. Došlý, "Perturbations of the half-linear Euler-Weber type differential equation," Journal of Mathematical Analysis and Applications, vol. 323, no. 1, pp. 426-440, 2006.

[4] S. Fišnarová and R. Mař́k, "Half-linear ODE and modified Riccati equation: comparison theorems, integral characterization of principal solution," Nonlinear Analysis: Theory, Methods and Applications, vol. 74, no. 17, pp. 6427-6433, 2011.

[5] Á. Elbert and A. Schneider, "Perturbations of the half-linear Euler differential equation," Results in Mathematics, vol. 37, no. 1-2, pp. 56-83, 2000.

[6] O. Došlý and M. Ünal, "Conditionally oscillatory half-linear differential equations," Acta Mathematica Hungarica, vol. 120, no. 1-2, pp. 147-163, 2008.

[7] N. Kandelaki, A. Lomtatidze, and D. Ugulava, “On oscillation and nonoscillation of a second order half-linear equation," Georgian Mathematical Journal, vol. 7, no. 2, pp. 329-346, 2000.

[8] O. Došlý, "A remark on the linearization technique in half-linear oscillation theory," Opuscula Mathematica, vol. 26, no. 2, pp. 305-315, 2006.

[9] O. Došlý and S. Fišnarová, "Half-linear oscillation criteria: perturbation in term involving derivative," Nonlinear Analysis: Theory, Methods \& Applications, vol. 73, no. 12, pp. 3756-3766, 2010.

[10] O. Došlý and M. Ünal, "Half-linear differential equations: linearization technique and its application," Journal of Mathematical Analysis and Applications, vol. 335, no. 1, pp. 450-460, 2007. 


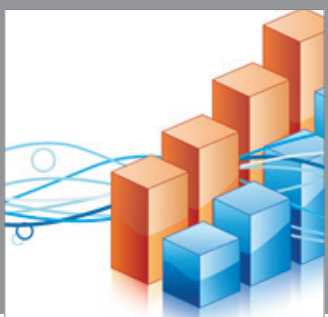

Advances in

Operations Research

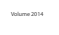

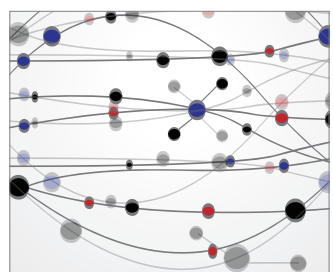

\section{The Scientific} World Journal
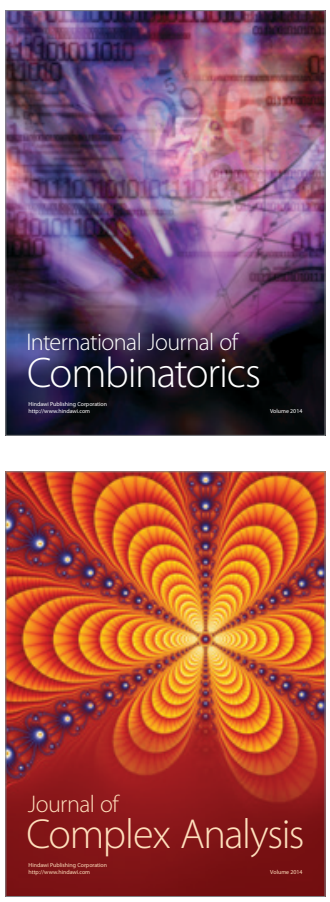

International Journal of

Mathematics and

Mathematical

Sciences
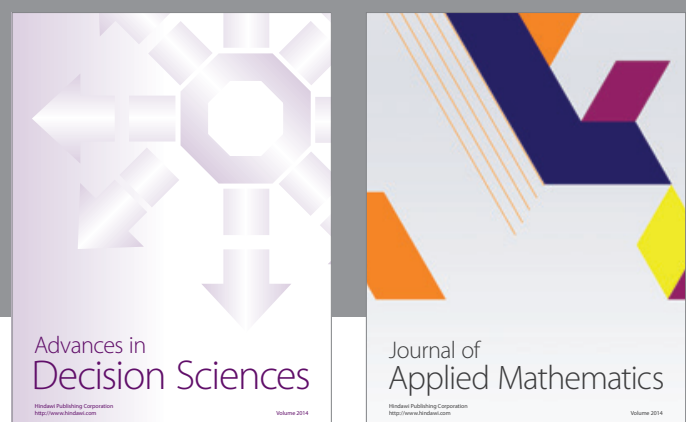

Journal of

Applied Mathematics
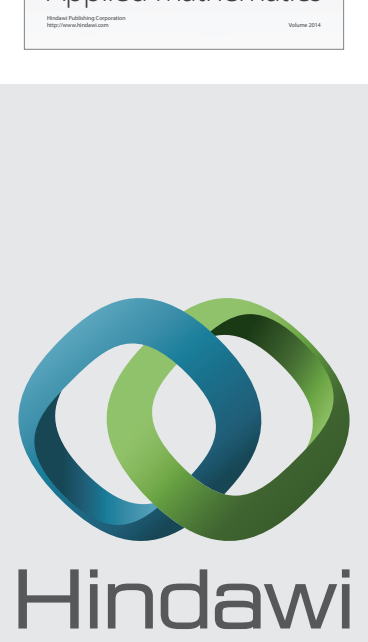

Submit your manuscripts at http://www.hindawi.com
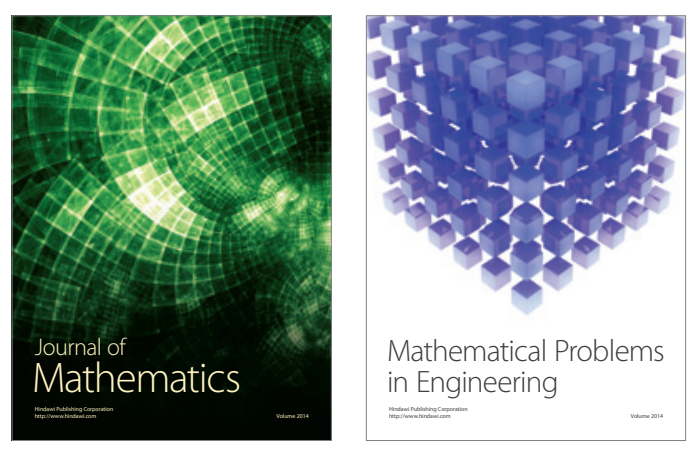

Mathematical Problems in Engineering
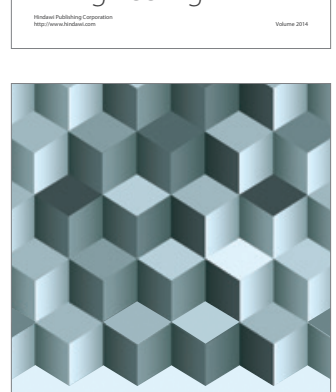

Journal of

Function Spaces
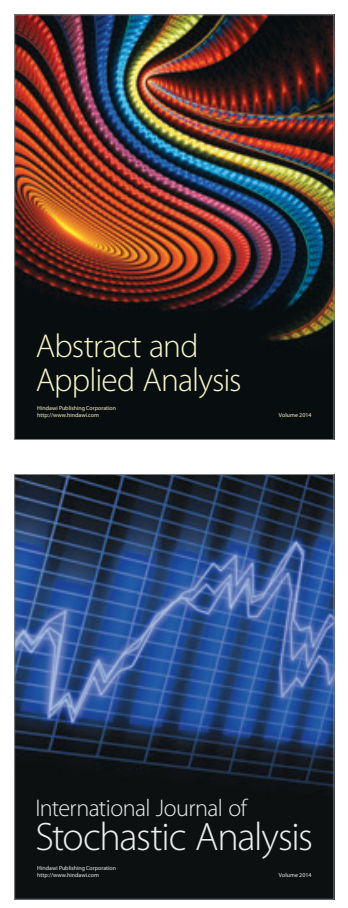

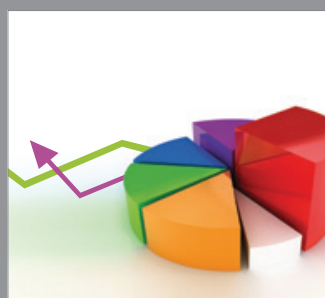

ournal of

Probability and Statistics

Promensencen
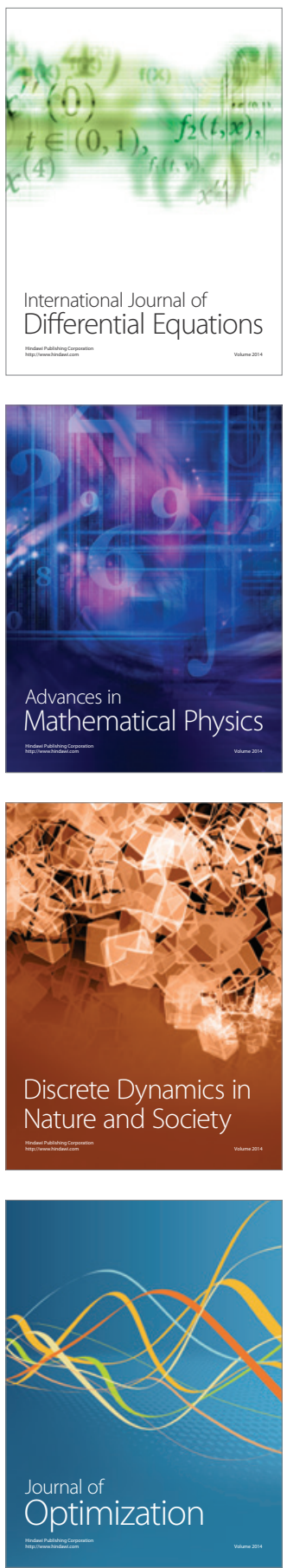